\title{
Fuel Sources and Carbon Dioxide Emissions by Electric Power Plants in the United States ${ }^{1}$
}

Alan W. Hodges and Mohammad Rahmani²

\section{Introduction}

The issue of global warming has drawn more attention in recent years due to rising sea levels, glacier retreat, Arctic shrinking, and altered patterns of agriculture (National Geographic 2007). Human activities add to global warming through carbon dioxide released by using coal, petroleum, natural gas, and other sources to produce energy for human consumption (IPCC 2007). Global warming has become an issue of concern because of its negative effects on the environment, human health, and economic well-being of all the people of the world (U.S. EPA 2008). The Intergovernmental Panel on Climate Change (IPCC) has set the goal of reducing carbon dioxide to $350 \mathrm{ppm}$ (parts per million) by 2025 (EEA 2008). Nations and the international community as a whole are trying to bring the rate of increase in emissions of greenhouse gases under control, or to mitigate the effects of such emissions through carbon capture and storage (sequestration) technologies. Renewable Portfolio Standard (RPS) and Renewable Fuel Standard (RFS) policies adopted by many states are efforts to mitigate carbon dioxide emissions (USDOE/EIA 2008). RPS requires electric power facilities to use a minimum percentage of their fuel from renewable sources, and RFS requires blending of renewable fuels (ethanol) with gasoline at specified minimum levels.

Carbon dioxide $\left(\mathrm{CO}_{2}\right)$ accounted for nearly 85 percent of total greenhouse gas emissions in 2006, with the balance from methane $\left(\mathrm{CH}_{4}\right)$, nitrogen oxides $\left(\mathrm{NO}_{2}\right)$, and fluorocarbons (HFC, PFC, and $\mathrm{SF}_{6}$ ). Electric power generation contributed almost 41 percent of the $\mathrm{CO}_{2}$ emissions from fossil fuels in the United States and 34 percent of all greenhouse gas emissions, the largest share of all major sectors of the U.S. economy, as shown in Table 1 (U.S. EPA 2008). Most electric power plants utilize coal and natural gas as the principal fuels. However, some are beginning to use non-polluting energy sources (e.g., sun, wind, and hydropower) and biomass fuels (which have little or no net emissions over the life cycle of feedstock growth and bio-fuel use) to generate electricity.

This report summarizes trends in carbon dioxide emissions of electric power plants by type of fuel and by state in the United States during the 2003-2007 period.

1. This is EDIS document FE796, a publication of the Food and Resource Economics Department, Florida Cooperative Extension Service, Institute of Food and Agricultural Sciences, University of Florida, Gainesville, FL. Published March 2009. Please visit the EDIS website at http://edis.ifas.ufl.edu.

2. Alan W. Hodges, extension scientist, and Mohammad Rahmani, coordinator of economic analysis, Food and Resource Economics Department, Florida Cooperative Extension Service, Institute of Food and Agricultural Sciences, University of Florida, Gainesville, FL.

The Institute of Food and Agricultural Sciences (IFAS) is an Equal Opportunity Institution authorized to provide research, educational information and other services only to individuals and institutions that function with non-discrimination with respect to race, creed, color, religion, age, disability, sex, sexual orientation, marital status, national origin, political opinions or affiliations. U.S. Department of Agriculture, Cooperative Extension Service, University of Florida, IFAS, Florida A. \& M. University Cooperative Extension Program, and Boards of County Commissioners Cooperating. Millie Ferrer, Interim Dean 


\section{Data}

This analysis uses monthly data, summarized annually for 2003-2007, on fuel consumption and electricity generation by electric power plants and industrial heat and power systems in the United States. The data are collected and published by the U.S. Department of Energy, Energy Information Administration (USDOE/EIA). Carbon dioxide uncontrolled emission factors are used to estimate the annual volume of carbon dioxide emissions by type of fuel used for electricity generation. Emission factors are the amount of carbon dioxide emitted per unit energy generated (pounds per million Btu/MMBtu) for each fuel type, based on EIA and Environmental Protection Agency (U.S. EPA) estimates. The most recent emission factors available from the 2000-2007 U.S. EPA studies were used for this analysis. The emission factor for mixed biogenic and non-biogenic municipal solid waste was adjusted upward based on U.S. EPA estimates from a 1997 study which showed that municipal solid waste in the United States contained 15.9 percent plastics. Table 2 shows the carbon dioxide emission factors by fuel groups and specific energy sources. Of course, nuclear and most renewable energy sources do not have direct $\mathrm{CO}_{2}$ emissions. While biomass fuels do emit $\mathrm{CO}_{2}$ upon combustion, the carbon is reabsorbed by growing plants over the lifecycle of biofuel production. However, direct emissions from biomass combustion are included in this analysis.

\section{Electricity Generation and Fuel Types}

The total electricity generation by electric power plants in the United States, net of internal uses, was about 4.2 gigawatt-hours ( $\mathrm{GWh}$, billion watt-hours) in 2007, as shown in Table 3. Three major fuel groups were used by electric power plants to generate electricity: coal, at over 48 percent, or more than 2 billion watt-hours; natural gas and propane, at over 21 percent, or nearly 900 million watt-hours; and nuclear energy, at 19 percent, or more than 800 million watt-hours. Renewable sources of energy, such as solar, geothermal, water, and wind, generated a total of 291 million watt-hours, or 7 percent of total electricity, and biomass generated 64 million watt-hours, or 1.5 percent, to the net electricity generation in the United States in 2007 (Table 3).

Trends in electricity generation for each energy source during the 2003-2007 period are summarized in Table 3, and charted for major fuel groups in Figure 1. Total electricity generation increased by about 7 percent between 2003 and 2007. Generation from natural gas and propane showed a significant increase of more than 37 percent during this time. Generation from all non-biomass renewable energy sources, such as solar, water, wind, and geothermal, which result in no or minimal emissions of carbon dioxide, actually decreased during this period. However, use of solar energy to produce electricity increased by more than 13 percent during the 2003-2007 period. Wind is now the fastest growing source of electricity due to substantial investments, particularly in the Great Plains region of the United States, with generation increasing by almost threefold over the last five years. Major efforts to promote greater use of biomass fuels for electricity generation have resulted in this source increasing by over 7 percent. It should be noted that biomass is considered carbon neutral because the carbon released during combustion is recaptured through plant growth. Woody biomass captures carbon from the atmosphere, whereas fossil fuels transfer underground carbon to the atmosphere (Biomass Energy Resource Center 2008). Higher petroleum prices have negatively affected the use of this fuel for generating electricity, decreasing its share by 51 percent since 2003, and it now represents only a little more than 1 percent of all fuel types used for electricity in 2007.

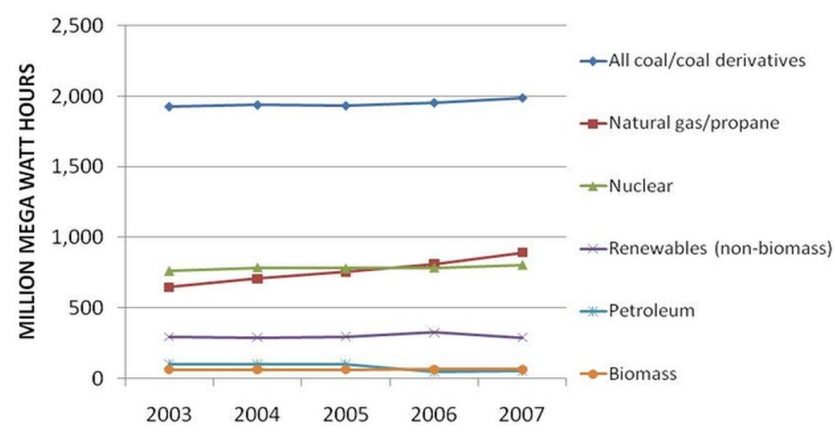

Figure 1. Net electricity generation by U.S. power plants by major fuel groups, 2003-2007. 


\section{Carbon Dioxide Emissions and Fuel Types}

The total carbon dioxide emissions by electric power plants in the United States exceeded 2.5 billion metric tons (2.756 Bn tons) in 2007 (Table 4). The largest fuel groups - coal, natural gas, and propane-dominated carbon dioxide emissions, over 94 percent of the total in 2007. Coal alone represented 78 percent of all $\mathrm{CO}_{2}$ emissions by electric power plants, or nearly 2 billion metric tons in 2007.

While total carbon dioxide emissions by power plants increased by 5 percent between 2003 and 2007, various fuel types showed different trends during this period. Bituminous coal, the highest contributor to power plant $\mathrm{CO}_{2}$ emissions in 2007 (38\%), showed a slight decrease of 1.6 percent when compared to 2003, whereas sub-bituminous coal, the second largest contributor to $\mathrm{CO}_{2}$ emissions, registered an almost 9 percent increase between 2003 and 2007. Lignite coal, with only a 3.5 percent share of $\mathrm{CO}_{2}$ emissions in 2007, decreased its contribution towards $\mathrm{CO}_{2}$ emissions by more than 10 percent. At the same time, the share of emissions from coal-based synfuels increased by more than 26 percent. Natural gas showed an increase in $\mathrm{CO}_{2}$ emissions of more than 34 percent between 2003 and 2007. Biomass accounted for only 2.7 percent of $\mathrm{CO}_{2}$ emissions in 2007 , but its share of carbon dioxide emissions decreased by 6 percent between 2003 and 2007. Figure 2 illustrates the trend during the 2003-2007 period in $\mathrm{CO}_{2}$ emissions for the major fuel groups.

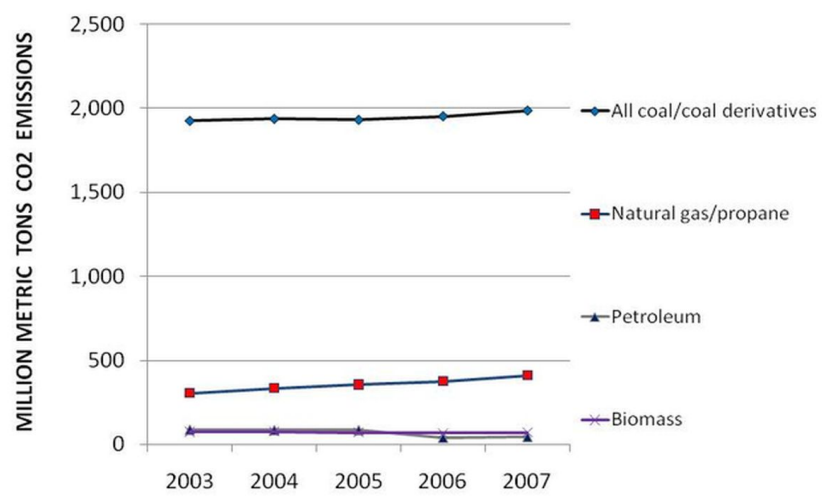

Figure 2. $\mathrm{CO}_{2}$ emissions by U.S. power plants by major fuel groups, 2003-2007.

\section{Emission Ratios}

The emission ratio represents the amount of $\mathrm{CO}_{2}$ emitted per unit of electricity generated (metric tons $\mathrm{CO}_{2}$ per megawatt-hour). This relationship was used to compare $\mathrm{CO}_{2}$ emissions by electric power plants across the United States, irrespective of differences in area, population, and other characteristics. Emission ratios are shown in Table 5 by state for 2007 and 2003 and the percentage change during this period. Power plants in states with lower emission ratios used more environmentally friendly fuel sources than those with higher emission ratios. Vermont and Washington had the lowest emission ratios of 0.09 and 0.13 , respectively, in 2007, and the highest decrease in emission ratios during the 2003-2007 period. Wyoming and North Dakota had the highest emission ratios of 0.99 and 1.02, respectively (Table 5).

\section{Conclusions}

Coal contributed over 78 percent of the total carbon dioxide emissions by electric power plants, but generated less than half $(48.6 \%)$ of all the electricity generated by U.S. power plants in 2007. Natural gas showed significantly lower $\mathrm{CO}_{2}$ emissions for the electricity it generated ( $16 \%$ of $\mathrm{CO}_{2}$ emissions for $21.5 \%$ of the generated electricity). Biomass fuels produced only 0.5 percent of the electricity in 2007and caused 2.7 percent of the $\mathrm{CO}_{2}$ emissions. Non-biomass renewable fuel sources generated 7 percent of the electricity and produced less than 0.1 percent of carbon dioxide emissions. Nuclear energy generated a significant share of electricity (19.4\%). However, the claim that nuclear energy does not cause any $\mathrm{CO}_{2}$ emissions has been challenged by the fact that extraction, production, and processing of fissile fuels (uranium, plutonium, and thorium) indirectly involve greenhouse gas emitting industries (Fritsche 1997). While the trend for electricity generation between 2003 and 2007 showed a 37 percent increase in natural gas usage, the use of non-polluting energy sources (e.g., sun, wind, and hydropower) registered a 1.1 percent decrease during the same time period. A 183 percent increase in wind energy usage during the 2003-2007 period is evidence of the potential for wind energy, although its share in electricity generation is still very small (0.8 percent). 
A recent report by the International Energy Agency estimated that $\$ 45$ trillion is needed to cut world carbon emissions in half by 2050 (Kanter 2008). Certainly, a major portion of this amount will be spent on low- or non- $\mathrm{CO}_{2}$ emitting sources of energy. The application of new technologies for using solar energy and an increased focus on wind energy for electricity generation show great promise in reducing $\mathrm{CO}_{2}$ emissions. States such as Nebraska, Nevada, New York, Oklahoma, Vermont, and Washington have reduced their emission ratios by over 10 percent since 2003 using energy sources with lower $\mathrm{CO}_{2}$ emissions.

To reduce greenhouse gas emissions, many states have adopted a Renewable Portfolio Standard policy requiring electric power facilities to reach a specified minimum percentage of their fuels from renewable resources by a certain date (USDOE/EIA 2007). Presently, 30 states (including the District of Columba) have adopted a mandatory Renewable Portfolio Standard (RPS), and four states have voluntary goals. The mandated goals for renewable resource use range from 4 percent in Massachusetts in 2009 to 25 percent in Illinois, Minnesota, and Oregon in 2025 (USDOE/EIA 2007). It is assumed that the use of renewable energy resources will reduce greenhouse gas emissions. Renewable Portfolio Standards may bring economic and health benefits such as job creation and cleaner air in addition to reducing greenhouse gas emissions (PEW Center 2008).

\section{References}

Bioenergy Resource Center. 2008. Biomass emissions: Air emissions from modern wood energy systems. Biomass Energy Resource Center, Montpelier, VT. http://www.biomasscenter.org/resources/fact-sheets/ fse-biomass-emissions.html

EEA. 2008. Climate change targets: 350 ppm and the EU two-degree target. European Environment Agency, Copenhagen, Denmark (June 23). http://www.eea.europa.eu/highlights/climate-changetargets-350-ppm-and-the-eu-2-degree-target

Fritsche, Uwe R. 1997. Comparing greenhouse-gas emissions and abatement costs of nuclear and alternative energy options from a life-cycle perspective. Paper presented at the CNIC Conference on Nuclear Energy and Greenhouse-Gas Emissions, Tokyo, Japan (November).

Kanter, James. 2008. Nations urged to spend \$45 trillion to battle carbon emission. International Herald Tribune, June 6. http://www.iht.com/articles/2008/06/06/business/ energy.php

IPCC. 2007. Climate Change 2007. Synthesis Report to Intergovernmental Panel on Climate Change, Valencia, Spain (November). http://www.ipcc.ch/pdf/assessment-report/ar4/syr/ ar4_syr.pdf

National Geographic. 2007. Effects of Global Warming. http://science.nationalgeographic.com/science/ environment/global-warming/gw-effects.html

PEW Center. 2008. Global Climate Change. Renewable Portfolio Standards (RPS). PEW Center, Arlington, VA (May).

http://www.pewclimate.org/what_s_being_done/ in_the_states/rps.cfm

U.S. DOE/EIA. 2008., EIA-906/920 Fuel Stock Data at Electric Power Generating Facilities, 2003-2007. U.S. Department of Energy, The Energy Information Administration, Washington, D.C. http://www.eia.doe.gov/cneaf/electricity/page/ eia906_920.html

\section{U.S. DOE/EIA. 2009. Carbon Dioxide} Uncontrolled Emission Factor, Electric Power Annual with Data for 2007. U.S. Department of Energy, Energy Information Administration, Washington, D.C. (January). http://www.eia.doe.gov/cneaf/electricity/epa/ epata3.html

U.S. DOE/EIA. 2007. States with Renewable Portfolio Standard. U.S. Department of Energy, Energy Information Administration, Washington, D.C. (June). http://www.eere.energy.gov/states/maps/ $\underline{\text { renewable_portfolio_states.cfm }}$ 
U.S. EPA. 2008. Climate Change, Basic

Information. U.S. Environmental Protection Agency, Washington, D.C. (April 1).

http://www.epa.gov/climatechange/basicinfo.html

U.S. EPA. 2007. Renewable Fuel Standard

Implementation: Frequently Asked Consumer

Questions. EPA420-F-07-062, U.S. Environmental

ProtectionAgency, Washington, D.C. (November).

http://www.epa.gov/oms/renewablefuels/

$\underline{420 \mathrm{f} 07062 . \mathrm{htm}}$

U.S. EPA. 2008. U.S. Greenhouse Gas

Inventory Reports-Trends in Greenhouse Gas

Emissions. U.S. Environmental Protection Agency, Washington, D.C. (April 10).

http://www.epa.gov/climatechange/emissions/

usinventoryreport.html 
Table 1. Greenhouse gas emissions by major sectors of the U.S. economy, 2006.

\begin{tabular}{|lrr|}
\hline \hline Sector & Emissions & Percent of Total \\
\hline & $\left(\mathrm{Tg} \mathrm{CO}_{2}\right.$ equivalent $)$ & $(\%)$ \\
\cline { 2 - 3 } Electric Power & 2,338 & 34 \\
Transportation & 1,970 & 28 \\
Industry & 1,372 & 20 \\
Agriculture & 534 & 8 \\
Commercial & 395 & 6 \\
Residential & 345 & 5 \\
Total & 6,952 & 100 \\
\hline Source: U.S. Environmental Protection Agency (2008). & \\
\hline \hline
\end{tabular}

Table 2. Carbon dioxide emission factors for electric power generation by fuel types in the United States.

\begin{tabular}{|llr|}
\hline \hline Fuel Group & Energy Source & Pounds Per Million Btu \\
\hline Biomass & Agriculture Crop Byproducts & 195.0 \\
& Black Liquor & 195.0 \\
& Landfill Gas & 115.1 \\
& Municipal Solid Waste (mixed biogenic \& non-biogenic) & 179.6 \\
& Municipal Solid Waste (non-biogenic) & 195.0 \\
& Other Biomass Gases & 115.1 \\
& Other Biomass Liquid (ethanol, fish oil) & 115.1 \\
Other Biomass Solid (animal manure; waste) & 195.0 \\
Sludge Waste & 199.5 \\
Wood Waste Liquids (red liquor; sludge wood) & 195.0 \\
Wood Waste Solids (paper; pellets; railroad ties; utility poles) & 195.0 \\
\hline Bituminous and Anthracite Coal & 205.3 \\
Lignite Coal & 215.4 \\
Coal-Based Synfuel (briquettes; pellets; extrusions) & 205.3 \\
Sub-Bituminous Coal & 212.7 \\
\hline Coal & & 205.3 \\
\hline \hline
\end{tabular}


Fuel Sources and Carbon Dioxide Emissions by Electric Power Plants in the United States

Table 2. Carbon dioxide emission factors for electric power generation by fuel types in the United States.

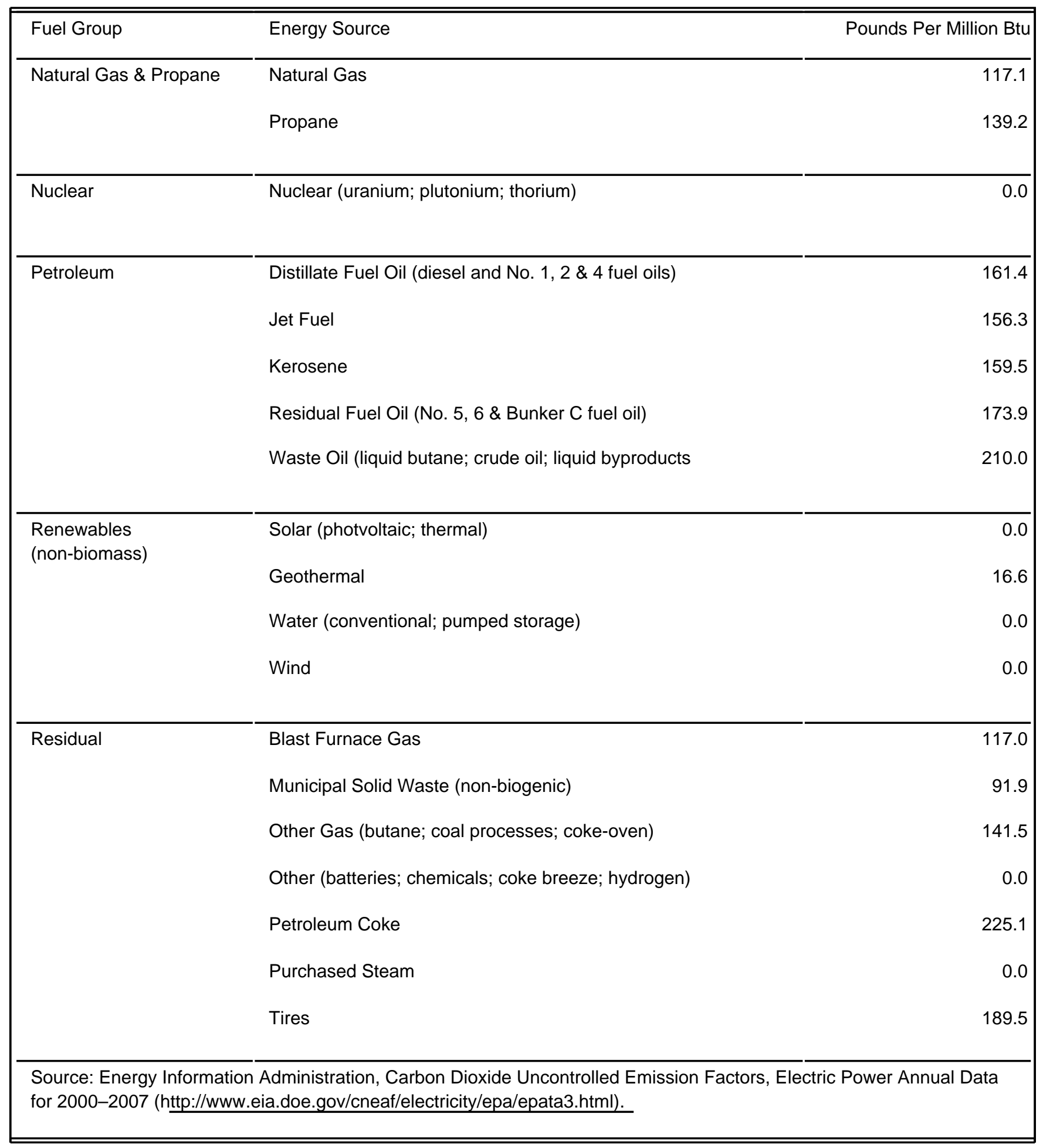


Table 3. Net electricity generation by energy source in the United States, 2003-2007.

\begin{tabular}{|c|c|c|c|c|c|c|c|}
\hline \multirow[t]{2}{*}{$\begin{array}{l}\text { Fuel Group and } \\
\text { Energy Source }\end{array}$} & 2003 & 2004 & 2005 & 2006 & 2007 & $\begin{array}{r}\text { Change } \\
2003-07\end{array}$ & $\begin{array}{r}\text { Share } \\
2007\end{array}$ \\
\hline & \multicolumn{5}{|c|}{ (gigawatt-hours) } & (\%) & (\%) \\
\hline Coal & $1,973,737$ & $1,978,620$ & $2,013,179$ & $1,990,926$ & $2,020,572$ & 2.4 & 48.6 \\
\hline $\begin{array}{l}\text { Bituminous and } \\
\text { Anthracite Coal }\end{array}$ & $1,063,588$ & $1,022,139$ & $1,042,601$ & $1,039,727$ & $1,040,008$ & -2.2 & 25.0 \\
\hline Lignite Coal & 91,834 & 88,973 & 91,054 & 88,382 & 82,894 & -9.7 & 2.0 \\
\hline $\begin{array}{l}\text { Coal-Based } \\
\text { Synfuel }\end{array}$ & 55,508 & 71,339 & 70,241 & 57,287 & 73,135 & 31.8 & 1.8 \\
\hline $\begin{array}{l}\text { Sub-Bituminous } \\
\text { Coal }\end{array}$ & 753,820 & 785,637 & 796,645 & 791,639 & 810,150 & 7.5 & 19.5 \\
\hline $\begin{array}{l}\text { Waste / Other } \\
\text { Coal }\end{array}$ & 8,987 & 10,531 & 12,639 & 13,890 & 14,385 & 60.1 & 0.3 \\
\hline $\begin{array}{l}\text { Natural Gas \& } \\
\text { Propane }\end{array}$ & 649,924 & 708,875 & 757,989 & 813,061 & 893,244 & 37.4 & 21.5 \\
\hline Natural Gas & 649,908 & 708,853 & 757,974 & 813,044 & 893,211 & 37.4 & 21.5 \\
\hline Propane & 16 & 21 & 15 & 17 & 32 & 101.4 & 0.0 \\
\hline $\begin{array}{l}\text { Nuclear (uranium; } \\
\text { plutonium; thorium) }\end{array}$ & 763,733 & 788,528 & 781,986 & 787,219 & 806,487 & 5.6 & 19.4 \\
\hline $\begin{array}{l}\text { Renewable } \\
\text { (non-biomass) }\end{array}$ & 294,514 & 290,275 & 297,441 & 326,492 & 291,171 & -1.1 & 7.0 \\
\hline Solar & 534 & 575 & 550 & 508 & 606 & 13.5 & 0.0 \\
\hline Geothermal & 14,424 & 14,811 & 14,692 & 14,568 & 14,839 & 2.9 & 0.4 \\
\hline Water & 267,271 & 259,929 & 263,763 & 282,689 & 241,319 & -9.7 & 5.8 \\
\hline Wind & 11,187 & 14,144 & 17,811 & 26,589 & 32,143 & 187.3 & 0.8 \\
\hline Biomass & 62,140 & 61,749 & 62,714 & 63,425 & 64,073 & 3.1 & 1.5 \\
\hline $\begin{array}{l}\text { Agricultue Crop } \\
\text { Byproducts }\end{array}$ & 725 & 747 & 675 & 661 & 726 & 0.2 & 0.0 \\
\hline Black Liquor & 18,660 & 17,782 & 18,230 & 18,268 & 18,231 & -2.3 & 0.4 \\
\hline Landfill Gas & 5,077 & 5,128 & 5,135 & 5,677 & 6,200 & 22.1 & 0.1 \\
\hline $\begin{array}{l}\text { Municipal Solid } \\
\text { Waste }\end{array}$ & 15,102 & 14,824 & 14,883 & 15,137 & 15,234 & 0.9 & 0.4 \\
\hline $\begin{array}{l}\text { Other Biomass } \\
\text { Gases }\end{array}$ & 1,088 & 840 & 758 & 788 & 854 & -21.4 & 0.0 \\
\hline $\begin{array}{l}\text { Other Biomass } \\
\text { Liquid }\end{array}$ & 8 & 12 & 7 & 10 & 11 & 44.4 & 0.0 \\
\hline $\begin{array}{l}\text { Other Biomass } \\
\text { Solid }\end{array}$ & 345 & 408 & 369 & 315 & 296 & -14.3 & 0.0 \\
\hline
\end{tabular}


Table 3. Net electricity generation by energy source in the United States, 2003-2007.

\begin{tabular}{|c|c|c|c|c|c|c|c|}
\hline \multirow[t]{2}{*}{$\begin{array}{l}\text { Fuel Group and } \\
\text { Energy Source }\end{array}$} & 2003 & 2004 & 2005 & 2006 & 2007 & $\begin{array}{l}\text { Change } \\
2003-07\end{array}$ & $\begin{array}{l}\text { Share } \\
2007\end{array}$ \\
\hline & \multicolumn{5}{|c|}{ (gigawatt-hours) } & $(\%)$ & (\%) \\
\hline Sludge Waste & 263 & 209 & 200 & 181 & 229 & -12.9 & 0.0 \\
\hline $\begin{array}{l}\text { Wood Waste } \\
\text { Liquids }\end{array}$ & 647 & 315 & 197 & 154 & 72 & -88.8 & 0.0 \\
\hline $\begin{array}{l}\text { Wood Waste } \\
\text { Solids }\end{array}$ & 18,222 & 19,479 & 20,254 & 20,227 & 20,212 & 10.9 & 0.5 \\
\hline Petroleum & 102,734 & 100,040 & 100,095 & 44,655 & 49,956 & -51.4 & 1.2 \\
\hline $\begin{array}{l}\text { Distillate Fuel } \\
\text { Oil }\end{array}$ & 15,169 & 10,660 & 11,198 & 7,236 & 8,423 & -44.5 & 0.2 \\
\hline Jet Fuel & 60 & 277 & 338 & 302 & 597 & 902.3 & 0.0 \\
\hline Kerosene & 439 & 422 & 517 & 185 & 482 & 9.8 & 0.0 \\
\hline $\begin{array}{l}\text { Residual Fuel } \\
\text { Oil }\end{array}$ & 85,940 & 87,551 & 87,189 & 36,054 & 39,561 & -54.0 & 1.0 \\
\hline Waste Oil & 1,127 & 1,129 & 853 & 877 & 893 & -20.7 & 0.0 \\
\hline Residual & 39,504 & 45,289 & 44,648 & 49,731 & 44,948 & 13.8 & 1.8 \\
\hline $\begin{array}{l}\text { Blast Furnace } \\
\text { Gas }\end{array}$ & 2,826 & 3,513 & 3,689 & 3,574 & 3,383 & 19.7 & 0.1 \\
\hline $\begin{array}{l}\text { Municipal Solid } \\
\text { Waste } \\
\text { (non-biogenic) }\end{array}$ & * & * & * & 6,661 & 6,666 & ** & 0.2 \\
\hline Other Gas & 12,758 & 13,232 & 12,613 & 12,469 & 11,999 & -6.0 & 0.3 \\
\hline Other & 5,024 & 5,863 & 4,124 & 3,925 & 3,697 & -26.4 & 0.1 \\
\hline Petroleum Coke & 16,672 & 20,731 & 22,427 & 19,709 & 15,752 & -5.5 & 0.4 \\
\hline $\begin{array}{l}\text { Purchased } \\
\text { Steam }\end{array}$ & 1,097 & 816 & 625 & 2,139 & 2,264 & 106.3 & 0.1 \\
\hline Tires & 1,128 & 1,134 & 1,171 & 1,254 & 1,188 & 5.3 & 0.0 \\
\hline Total All Fuel Types & $3,883,185$ & $3,970,555$ & $4,055,423$ & $4,064,702$ & $4,159,514$ & 7.1 & $\overline{100.0}$ \\
\hline $\begin{array}{l}\text { Year-Over-Year } \\
\text { Percent Change }\end{array}$ & & 2.25 & 2.14 & 0.23 & 2.33 & & \\
\hline
\end{tabular}


Table 4. Carbon dioxide emissions by U.S. electric power plants by energy source, 2003-2007.

\begin{tabular}{|c|c|c|c|c|c|c|c|}
\hline \multirow[t]{2}{*}{$\begin{array}{l}\text { Fuel Group and } \\
\text { Energy Source }\end{array}$} & 2003 & 2004 & 2005 & 2006 & 2007 & $\begin{array}{r}\text { Change } \\
2003-07\end{array}$ & $\begin{array}{l}\text { Share } \\
2007\end{array}$ \\
\hline & \multicolumn{5}{|c|}{ (1,000 metric tons) } & $(\%)$ & (\%) \\
\hline Coal & $1,927,847$ & $1,939,598$ & $1,933,992$ & $1,954,332$ & $1,987,563$ & 3.1 & 78.1 \\
\hline $\begin{array}{l}\text { Bituminous and } \\
\text { Anthracite Coal }\end{array}$ & $1,002,095$ & 968,583 & 986,591 & 982,394 & 985,365 & -1.7 & 38.7 \\
\hline Lignite Coal & 100,958 & 94,349 & 52,427 & 95,244 & 90,281 & -10.6 & 3.5 \\
\hline $\begin{array}{l}\text { Coal-Based } \\
\text { Synfuel }\end{array}$ & 52,449 & 66,406 & 64,158 & 52,197 & 66,462 & 26.7 & 2.6 \\
\hline $\begin{array}{l}\text { Sub-Bituminous } \\
\text { Coal }\end{array}$ & 761,676 & 797,903 & 816,458 & 808,874 & 829,454 & 8.9 & 32.6 \\
\hline $\begin{array}{l}\text { Waste / Other } \\
\text { Coal }\end{array}$ & 10,669 & 12,358 & 14,359 & 15,623 & 16,001 & 50.0 & 0.6 \\
\hline $\begin{array}{l}\text { Natural Gas \& } \\
\text { Propane }\end{array}$ & 304,670 & 333,614 & 354,359 & 375,018 & 409,854 & 34.5 & 16.1 \\
\hline Natural Gas & 304,659 & 333,597 & 354,348 & 375,007 & 409,828 & 34.5 & 16.1 \\
\hline Propane & 11 & 17 & 11 & 11 & 26 & 134.6 & 0.0 \\
\hline Biomass & 73,967 & 75,700 & 70,677 & 66,292 & 69,462 & -6.1 & 2.7 \\
\hline $\begin{array}{l}\text { Agricultue Crop } \\
\text { Byproducts }\end{array}$ & 1,253 & 1,115 & 1,109 & 1,122 & 1,270 & 1.4 & 0.0 \\
\hline Black Liquor & 21,335 & 23,069 & 18,448 & 19,744 & 21,509 & 0.8 & 0.8 \\
\hline Landfill Gas & 3,439 & 3,644 & 3,483 & 4,001 & 4,426 & 28.7 & 0.2 \\
\hline $\begin{array}{l}\text { Municipal Solid } \\
\text { Waste }\end{array}$ & 21,932 & 22,207 & 22,052 & 13,679 & 13,935 & -13.2 & 0.7 \\
\hline $\begin{array}{l}\text { Other Biomass } \\
\text { Gases }\end{array}$ & 597 & 487 & 511 & 519 & 539 & -9.7 & 0.0 \\
\hline $\begin{array}{l}\text { Other Biomass } \\
\text { Liquid }\end{array}$ & 6 & 6 & 3 & 5 & 5 & -17.8 & 0.0 \\
\hline $\begin{array}{l}\text { Other Biomass } \\
\text { Solid }\end{array}$ & 479 & 671 & 528 & 507 & 510 & 6.4 & 0.0 \\
\hline Sludge Waste & 329 & 381 & 270 & 214 & 267 & -19.0 & 0.0 \\
\hline $\begin{array}{l}\text { Wood Waste } \\
\text { Liquids }\end{array}$ & 789 & 201 & 150 & 339 & 103 & -87.0 & 0.0 \\
\hline $\begin{array}{l}\text { Wood Waste } \\
\text { Solids }\end{array}$ & 23,808 & 23,920 & 24,024 & 26,161 & 26,897 & 13.0 & 1.1 \\
\hline
\end{tabular}


Table 4. Carbon dioxide emissions by U.S. electric power plants by energy source, 2003-2007.

\begin{tabular}{|c|c|c|c|c|c|c|c|}
\hline \multirow[t]{2}{*}{$\begin{array}{l}\text { Fuel Group and } \\
\text { Energy Source }\end{array}$} & 2003 & 2004 & 2005 & 2006 & 2007 & $\begin{array}{l}\text { Change } \\
2003-07\end{array}$ & $\begin{array}{l}\text { Share } \\
2007\end{array}$ \\
\hline & \multicolumn{5}{|c|}{ (1,000 metric tons) } & (\%) & (\%) \\
\hline Petroleum & 85,062 & 82,894 & 82,691 & 37,405 & 42,067 & -50.5 & 1.7 \\
\hline Distillate Fuel Oil & 12,604 & 8,789 & 9,000 & 5,685 & 7,049 & -44.1 & 0.3 \\
\hline Jet Fuel & 58 & 200 & 254 & 213 & 377 & 553.2 & 0.0 \\
\hline Kerosene & 354 & 374 & 421 & 168 & 412 & 16.5 & 0.0 \\
\hline Residual Fuel Oil & 71,178 & 72,493 & 72,275 & 30,554 & 33,474 & -53.0 & 1.3 \\
\hline Waste Oil & 868 & 1,038 & 742 & 784 & 755 & -13.0 & 0.0 \\
\hline Residual & 28,770 & 35,573 & 36,524 & 39,747 & 34,607 & 20.3 & 1.4 \\
\hline $\begin{array}{l}\text { Blast Furnace } \\
\text { Gas }\end{array}$ & 2,188 & 2,281 & 2,702 & 2,885 & 2,472 & 13.0 & 0.1 \\
\hline $\begin{array}{l}\text { Municipal Solid } \\
\text { Waste } \\
\text { (non-biogenic) }\end{array}$ & * & * & * & 5,065 & 5,105 & ** & 0.2 \\
\hline Other Gas & 7,400 & 9,215 & 8,077 & 8,124 & 7,662 & 3.5 & 0.3 \\
\hline Petroleum Coke & 18,040 & 22,835 & 24,447 & 22,203 & 17,936 & -0.6 & 0.7 \\
\hline Tires & 1,142 & 1,242 & 1,298 & 1,470 & 1,431 & 25.3 & 0.1 \\
\hline $\begin{array}{l}\text { Non-Biomass } \\
\text { Renewables / } \\
\text { Geothermal }\end{array}$ & 2,283 & 2,344 & 2,325 & 2,305 & 2,348 & 2.9 & 0.1 \\
\hline Total All Sources & $2,422,599$ & $2,469,723$ & $2,480,568$ & $2,475,098$ & $2,545,900$ & 5.1 & 100.0 \\
\hline $\begin{array}{l}\text { Annual Percent } \\
\text { Change }\end{array}$ & & 1.95 & 0.44 & -0.22 & 2.86 & & \\
\hline
\end{tabular}


Table 5. Emission ratios by U.S. power plants, 2003 and 2007, and percent change.

\begin{tabular}{|c|c|c|c|c|c|}
\hline State & $\begin{array}{r}\text { Electricity } \\
\text { Generation, } \\
2007\end{array}$ & $\begin{array}{l}\text { Carbon Dioxide } \\
\text { Emissions, } 2007\end{array}$ & $\begin{array}{r}\text { Emission Ratio, } \\
2003\end{array}$ & $\begin{array}{r}\text { Emission Ratio, } \\
2007\end{array}$ & $\begin{array}{r}\text { Change } \\
\text { Emission Ratio } \\
2003-07\end{array}$ \\
\hline & (gigawatt-hours) & $(1,000$ metric tons $)$ & $\begin{array}{l}\text { (metric tons per } \\
\text { megawatt-hour) }\end{array}$ & $\begin{array}{l}\text { (metric tons per } \\
\text { megawatt-hour) }\end{array}$ & (\%) \\
\hline Vermont & 6,382 & 576 & 0.14 & 0.09 & -37 \\
\hline Nevada & 31,888 & 16,615 & 0.70 & 0.52 & -26 \\
\hline Washington & 106,079 & 14,078 & 0.16 & 0.13 & -18 \\
\hline Oklahoma & 72,253 & 51,036 & 0.80 & 0.71 & -12 \\
\hline New York & 146,499 & 53,821 & 0.41 & 0.37 & -10 \\
\hline Nebraska & 32,957 & 20,866 & 0.71 & 0.63 & -10 \\
\hline lowa & 49,760 & 41,351 & 0.91 & 0.83 & -9 \\
\hline New Hampshire & 23,413 & 8,425 & 0.39 & 0.36 & -8 \\
\hline Virginia & 78,594 & 42,239 & 0.58 & 0.54 & -8 \\
\hline Florida & 225,832 & 132,280 & 0.63 & 0.59 & -7 \\
\hline New Mexico & 35,953 & 31,800 & 0.96 & 0.88 & -7 \\
\hline Connecticut & 33,494 & 10,953 & 0.35 & 0.33 & -6 \\
\hline New Jersey & 63,088 & 20,888 & 0.35 & 0.33 & -6 \\
\hline Kansas & 50,080 & 39,179 & 0.83 & 0.78 & -6 \\
\hline Colorado & 52,954 & 42,872 & 0.86 & 0.81 & -6 \\
\hline Massachusetts & 47,513 & 26,601 & 0.59 & 0.56 & -5 \\
\hline Minnesota & 53,611 & 36,949 & 0.73 & 0.69 & -5 \\
\hline Mississippi & 49,880 & 29,216 & 0.61 & 0.59 & -4 \\
\hline Wisconsin & 63,402 & 47,227 & 0.78 & 0.74 & -4 \\
\hline Ohio & 156,069 & 130,774 & 0.87 & 0.84 & -4 \\
\hline Utah & 43,691 & 38,355 & 0.91 & 0.88 & -4 \\
\hline Missouri & 91,147 & 77,281 & 0.87 & 0.85 & -3 \\
\hline Wyoming & 45,581 & 45,180 & 1.03 & 0.99 & -3 \\
\hline Rhode Island & 7,049 & 2,997 & 0.43 & 0.42 & -2 \\
\hline Arkansas & 55,074 & 30,839 & 0.57 & 0.56 & -2 \\
\hline Pennsylvania & 227,278 & 131,161 & 0.59 & 0.58 & -2 \\
\hline Texas & 405,582 & 258,103 & 0.65 & 0.64 & -1 \\
\hline Maryland & 49,968 & 31,159 & 0.63 & 0.62 & -1 \\
\hline Montana & 28,491 & 20,199 & 0.71 & 0.71 & 0 \\
\hline Arizona & 113,022 & 55,854 & 0.49 & 0.49 & 0 \\
\hline
\end{tabular}


Table 5. Emission ratios by U.S. power plants, 2003 and 2007, and percent change.

\begin{tabular}{|c|c|c|c|c|c|}
\hline State & $\begin{array}{r}\text { Electricity } \\
\text { Generation, } \\
2007\end{array}$ & $\begin{array}{l}\text { Carbon Dioxide } \\
\text { Emissions, } 2007\end{array}$ & $\begin{array}{r}\text { Emission Ratio, } \\
2003\end{array}$ & $\begin{array}{r}\text { Emission Ratio, } \\
2007\end{array}$ & $\begin{array}{r}\text { Change } \\
\text { Emission Ratio } \\
2003-07\end{array}$ \\
\hline & $\overline{\text { (gigawatt-hours) }}$ & $(1,000$ metric tons $)$ & $\begin{array}{l}\text { (metric tons per } \\
\text { megawatt-hour) }\end{array}$ & $\begin{array}{l}\text { (metric tons per } \\
\text { megawatt-hour) }\end{array}$ & (\%) \\
\hline Maine & 15,660 & 8,348 & 0.53 & 0.53 & 0 \\
\hline Louisiana & 92,766 & 54,669 & 0.59 & 0.59 & 0 \\
\hline Michigan & 120,282 & 80,972 & 0.67 & 0.67 & 0 \\
\hline West Virginia & 93,940 & 84,886 & 0.90 & 0.90 & 0 \\
\hline Indiana & 130,728 & 124,119 & 0.94 & 0.95 & 1 \\
\hline North Dakota & 30,820 & 31,573 & 1.02 & 1.02 & 1 \\
\hline Idaho & 11,319 & 2,044 & 0.18 & 0.18 & 2 \\
\hline Kentucky & 97,477 & 93,899 & 0.94 & 0.96 & 2 \\
\hline Illinois & 200,332 & 101,608 & 0.49 & 0.51 & 3 \\
\hline Alabama & 144,575 & 91,482 & 0.61 & 0.63 & 4 \\
\hline Hawaii & 11,714 & 9,703 & 0.80 & 0.83 & 4 \\
\hline Georgia & 145,394 & 97,958 & 0.64 & 0.67 & 5 \\
\hline Delaware & 8,510 & 7,836 & 0.88 & 0.92 & 5 \\
\hline South Carolina & 103,911 & 44,712 & 0.41 & 0.43 & 6 \\
\hline Tennessee & 94,930 & 57,854 & 0.58 & 0.61 & 6 \\
\hline California & 214,099 & 67,108 & 0.29 & 0.31 & 8 \\
\hline North Carolina & 130,239 & 79,783 & 0.57 & 0.61 & 8 \\
\hline Oregon & 53,578 & 11,197 & 0.99 & 0.21 & 9 \\
\hline Alaska & 6,888 & 4,128 & 0.55 & 0.60 & 9 \\
\hline South Dakota & 5,664 & 3,061 & 0.46 & 0.54 & 16 \\
\hline
\end{tabular}

